\title{
Nível de atividade física, estresse e saúde em bancários
}

\author{
M.S. Viana, A. Andrade, A.R. Back, D.I.C. Vasconcellos
}

O objetivo deste estudo foi investigar a relação entre nível de atividade física, estresse e saúde de bancários e bancárias. Participaram do estudo 283 bancários (56.6\% homens $/ 43.4 \%$ mulheres) com idade média de 40 anos. Os instrumentos utilizados foram: Questionário de Atividades Físicas Habituais (Pate et al., 1995); Escala de Estresse Percebido (Cohen, Karmack, \& Mermelsteinm, 1983); e duas escalas de saúde autoavaliada (Andrade, 2001). Os bancários mais ativos fisicamente mostraram-se menos estressados $(F=4.87, p=.008)$; resultado significativo apenas para as mulheres $(F=$ $4.11, p=.019)$. Os ativos também apresentaram melhor saúde percebida $(p=.000, \gamma=$ .56) e menor frequência de adoecimento $(p=.02, \gamma=-.34)$; resultados significativos somente para os homens ( $p=.001, \gamma=.60$ e $p=.033, \gamma=-.33$, respectivamente). Bancários com melhor saúde percebida foram significativamente menos estressados $(F=$ $13.45, p=.000)$; resultado significativo para homens $(F=9.75, p=.000)$ e mulheres $(F$ $=7.88, p=.000)$. Quem adoece menos esteve menos estressado $(F=5.40, p=.001)$; resultado significativo apenas para as bancárias $(F=4.60, p=.004)$. Os achados indicam existir relações entre as variáveis investigadas, e que tais relações não se dão de maneira equivalente para homens e mulheres dessa população.

Palavras-chave: atividade física, estresse, saúde, bancários

\section{Level of physical activity, stress and health of bank clerks}

The purpose of this study was to investigate the relationship among level of physical activity, stress and health in male and female bank clerks. Two hundred eighty three bank clerks answered the Questionnaire of Habitual Physical Activities (Pate et al., 1995), the Perceived Stress Scale (Cohen, Karmack, \& Mermelsteinm, 1983), and two scales of selfevaluated health (Andrade, 2001). More active bank clerks showed themselves as less stressed $(F=4.87, p=.008)$, significant results only for women $(F=4.11, p=.019)$. More active bank clerks also perceived themselves as healthier $(p=.000, \gamma=.56)$ and fell ill less frequently $(p=.02, \gamma=-.34)$, significant results only for men $(p=.001, \gamma=$ .60 e $p=.033, \gamma=-.33$, respectively). Bank clerks with better perceived health showed significantly lower stress $(F=13.45, p=.000)$, significant results for both men $(F=$ $9.75, p=.000)$ and women $(F=7.88, p=.000)$, as well as those getting sick less frequently $(F=5.40, p=.001)$, significant results only for women $(F=4.60, p=.004)$. Findings indicated a relationship among all the variables investigated and differing relationships for men and women.

Keywords: physical activity, stress, health, bank clerks

\footnotetext{
Maick da Silveira Viana, Alexandro Andrade, Alexandre Rodrigo Back e Diego Itibere Cunha Vasconcellos. Laboratório de Psicologia do Esporte e do Exercício - LAPE, Centro de Ciências da Saúde e do Esporte CEFID, Universidade do Estado de Santa Catarina - UDESC.

Endereço para correspondência: Maick da Silveira Viana, Laboratório de Psicologia do Esporte e do Exercício LAPE, Centro de Ciências da Saúde e do Esporte - CEFID, Rua Pascoal Simone, 358 - Coqueiros, CEP: 88080-350, Florianópolis - Santa Catarina, Brasil.

E-mail: efisica@gmail.com
} 
Nas duas últimas décadas tem crescido o interesse científico por pesquisas relacionadas ao estresse no trabalho. Segundo Paschoal e Tamayo (2004), uma das razões para o aumento do número de pesquisas sobre esse tema se deve ao impacto negativo do estresse ocupacional na saúde e no bem-estar dos trabalhadores e, conseqüentemente, no funcionamento e na efetividade das organizações. O estresse no trabalho está relacionado às situações onde a pessoa percebe o seu ambiente de trabalho como ameaçador as suas necessidades de realização pessoal e profissional e/ou a sua saúde física e mental. Tais situações prejudicam a interação do trabalhador com o trabalho e seu ambiente, na medida em que este contém demandas excessivas a ele, ou que ele não possui recursos adequados para enfrentar tais situações (França \& Rodrigues, 1997).

As primeiras investigações que associaram o estresse no trabalho a problemas de saúde datam da década de 1960. Essas pesquisas focaram-se especialmente em aspectos físicos da saúde, encontrando relações entre a ocorrência de doenças coronarianas e elevados níveis de estresse no trabalho. Porém, não apenas doenças de ordem física são relacionadas ao estresse laboral. Para Palácios, Duarte e Câmara (2002), quanto mais se procura compreender a relação entre estresse no trabalho e saúde, mais ganham destaque as questões ligadas à saúde mental.

Existem profissões que são reconhecidamente mais estressantes e conseqüentemente mais afetadas por doenças laborais, dentre estas está a profissão de bancário. Por essa característica, os bancários têm se tornado foco de importantes estudos relacionados à saúde ocupacional (Andrade, 2001; Palácios, Duarte, \& Câmara, 2002; Silva, Pinheiro, \& Sakurai, 2007; Souza, Messing, Menezes, \& Cho 2002), mostrando ser relevante o estudo do estresse e sua relação com o acometimento de doenças nessa população.

$\mathrm{Na}$ busca por alternativas que valorizem a qualidade de vida dos trabalhadores, uma das opções que vem sendo apontada como favorecedora de melhorias na saúde e redução do estresse é a atividade física ( $\mathrm{Hu}$ et al., 2004; Kouvonen et al., 2005). Tanto estudos transversais, como experimentais, têm apontado com certa consistência que pessoas, de populações diversas, são beneficiadas pela prática de atividades físicas (Nahas, 2001).

Apesar das pesquisas evidenciarem de forma consistente a relação entre nível de atividade física, estresse e saúde, percebe-se uma lacuna a ser preenchida quanto às diferenças existentes entre homens e mulheres para essa relação. Sabe-se que homens e mulheres diferenciam-se quanto aos níveis de saúde (Aquino, Menezes, \& Amoedo, 1992; McDonough \& Walters, 2001; Read \& Gorman, 2006), a incidência e causa de estresse (Calais, Andrade, \& Lipp, 2003; Lundberg, 2005; Lundberg \& Frankenhaeuser, 1999; Matud, 2004), os hábitos de atividade física (Wijndaele et al., 2007) e as demandas psicológicas no trabalho (Rocha \& Debert-Ribeiro, 2001), mas não se essas variáveis interagem de forma 
equivalente nos diferentes sexos. De acordo com Plotnikoff, Mayhew, Birkett, Loucaides e Fodor (2004), as pesquisas na área da atividade física e saúde são ainda limitadas, pois as possíveis diferenciações entre grupos devem ser mais aprofundadas. Algumas iniciativas que buscam melhor compreender as possíveis diferenças entre esses grupos têm sido tomadas recentemente (Kouvonen et al., 2005; Plotnikoff et al., 2004), porém os resultados são ainda pouco conclusivos.

De acordo com a problematização apresentada, este estudo teve o propósito de investigar a relação entre o nível de atividade física, o estresse e a saúde de bancários e bancárias.

\section{MÉTODO}

Trata-se de uma pesquisa transversal de campo, descritiva do tipo correlacional.

\section{Amostra}

Participaram do estudo 283 bancários de ambos os sexos ( $56.6 \%$ homens e $43.4 \%$ mulheres), trabalhadores do setor administrativo e de atendimento ao público de um banco estatal na região da Grande Florianópolis, Santa Catarina, Brasil.

\section{Instrumentos}

$\mathrm{O}$ instrumento utilizado para a coleta das informações foi o Questionário de Auto-Avaliação do Estilo de Vida, Ocorrência e Controle do Estresse, desenvolvido por Andrade (2001). Três instrumentos estão incluídos no questionário utilizado: o Questionário de Atividades Físicas Habituais (Pate et al., 1995); a Escala de Estresse Percebido (Cohen, Karmack, \& Mermelsteinm, 1983); e para investigar a saúde auto-avaliada duas escalas sobre a percepção subjetiva da saúde elaboradas por Andrade (2001).

A versão brasileira do Questionário de Atividades Físicas Habituais foi apresentada por Nahas (2001). A utilização de tal versão do instrumento tem demonstrado bons resultados em estudos realizados no Brasil (Adami, Frainer, Santos, Fernandes, \& De-Oliveira, 2008; Glaner, 2007). O Questionário de Atividades Físicas Habituais é composto por 11 questões referentes às atividades físicas habituais, sistematizadas e de lazer do participante. Após o somatório dos pontos obtidos nas questões, os bancários foram classificados como: inativos (< 6 pontos); moderadamente ativos (6 - 11 pontos); ativos (12 - 20 pontos); e muito ativos (> 21 pontos). Depois de uma análise sobre as diferenças teóricas que cada grupo poderia apresentar em comparação aos demais, e considerando a pequena representatividade de bancários muito ativos $(n=4)$, foi estabelecido que os grupos ativo e muito ativo formariam um grupo único, denominado grupo ativo.

Quanto à Escala de Estresse Percebido, foi utilizada a versão apresentada por Ururahy (1997), que obteve bons resultados no estudo de Andrade (2001). A escala é composta por 14 sub-escalas do tipo Likert, nas quais o participante assinala uma opção que varia de 0 a 4 para cada uma, onde: $0=$ nunca; 1 = pouco; 2 = às vezes; $3=$ regularmente; e $4=$ sempre. O resulta- 
do da escala - Índice de Estresse Percebido (ISP) - pode variar de 0 (sem estresse) a 56 (extremo estresse).

As duas questões destinadas à avaliação da saúde dos bancários são também escalas do tipo Likert. A primeira questiona a respeito da percepção do bancário sobre sua saúde, tendo opções de resposta que variam de 0 a 4 , onde: $0=$ péssima; $1=$ ruim; $2=$ regular; 3 $=$ boa; $\mathrm{e} 4=$ excelente. A segunda questiona a frequência com que o bancário fica doente, tendo opções de resposta também de 0 a 4 , onde: $0=$ não fico doente; 1 = fico doente poucas vezes; 2 = fico doente às vezes; $3=$ fico doente muitas vezes; e $4=$ sempre fico doente.

\section{Procedimentos}

Previamente à coleta dos dados, foi obtida autorização da gerência regional do banco investigado. Os instrumentos de pesquisa foram distribuídos aos bancários da região pesquisada, em seus locais de trabalho antes do início do expediente. Após explanação dos objetivos da pesquisa os bancários que se dispuseram a participar foram instruídos a responder o questionário preferencialmente em suas residências, sozinhos e em ambiente tranquilo. Nos dias seguintes à entrega dos instrumentos, os pesquisadores retornaram às agências para o recolhimento dos questionários, os quais totalizaram 315. Aproveitaram-se 283 para o estudo, sendo excluídos questionários incompletos, rasurados e com resultados discrepantes.

\section{Análise estatística}

Para o tratamento estatístico utilizou-se de estatística descritiva e inferencial. Quanto à estatística descritiva, realizou-se análise exploratória dos dados para: verificação da distribuição dos dados, por meio do teste de Kolmogorov-Smirnov e histogramas de freqüência, distribuição das freqüências e percentuais, análises das tendências centrais por meio de média, e dispersão dos dados com o desvio padrão.

Quanto à estatística inferencial, considerando a normalidade dos dados, os grupos foram comparados por meio de ANOVA com Post-Hoc de Scheffé, e teste t de Student. Utilizou-se ainda do teste Qui-Quadrado para verificar a associação de dados categóricos com coeficiente $\mathrm{V}$ de Cramér para verificação da força das associações entre variáveis nominais e coeficiente Gamma $(\gamma)$ para variáveis ordinais. $\mathrm{O} \alpha$ estabelecido foi de .05 como nível de significância $(p<.05)$.

Os dados foram tabulados e analisados no programa "Statistic Package for Social Sciences - SPSS" versão 13.0.

\section{Aspectos éticos}

Esta pesquisa foi submetida e aprovada pelo Comitê de Ética em Pesquisa em Seres Humanos da Universidade do Estado de Santa Catarina sob $n^{\circ}$ de referência 01/2006. Todas as normas e diretrizes regulamentadoras da pesquisa envolvendo serem humanos foram seguidas durante o transcorrer do estudo. 
Todos os participantes assinaram o Termo de Consentimento Livre e Esclarecido para participação no estudo, o qual convidava o bancário a participar da pesquisa e explicitava seu caráter voluntário e o anonimato de todas as informações obtidas pelos pesquisadores.

\section{RESULTADOS}

\section{Caracterização dos participantes}

Quase a totalidade dos participantes se declarou de etnia/raça branca (98.1\%), sendo a média de idade de 40 anos $(D P=6.8 ; \operatorname{Min}=19, \operatorname{Max}=57)$. Os homens apresentaram média de idade de 41 anos $(D P=6.9 ; \operatorname{Min}=19$, Max $=57)$, pouco superior a média das mulheres que foi de 40 anos $(D P=6.6$; $\operatorname{Min}=20, \operatorname{Max}=53)$. A maioria dos bancários $(78 \%)$ completou o ensino superior $(80.3 \%$ das mulheres e $75.9 \%$ dos homens). O tempo médio de trabalho no banco foi de 17.3 anos ( $D P=$ 7.3; $\operatorname{Min}=1, \operatorname{Max}=35$ ), sendo o tem- po de trabalho dos homens $(M=17.5$, $D P=7.5 ; \operatorname{Min}=1, \operatorname{Max}=35$ ) pouco superior ao das mulheres $(M=17.0$, $D P=7.1 ; \operatorname{Min}=1, \operatorname{Max}=32$ ). Esses resultados demonstram que homens e mulheres participantes do estudo se assemelham quanto às características gerais.

\section{Nível de atividade física}

A maioria dos participantes é inativo fisicamente. Bancários foram significativamente mais ativos do que as bancárias, tendo maior proporção de ativos e menor de inativos. Considerando o coeficiente $\mathrm{V}$, a associação entre nível de atividade física e sexo foi fraca (tabela 1).

\section{Nível de Estresse}

Quanto ao nível de estresse, homens e mulheres não se diferenciaram significativamente, tanto em relação a sua média quanto à dispersão dos dados, que são semelhantes (tabela 2).

Tabela 1

Nível de atividade física de bancários da amostra geral, homens e mulheres

\begin{tabular}{lccc}
\hline & Amostra Geral & Homens ${ }^{*}$ & Mulheres $^{*}$ \\
\hline Inativos & $173(61.2 \%)$ & $89(56.0 \%)$ & $82(67.2 \%)$ \\
Moderadamente Ativos & $68(24.0 \%)$ & $39(24.5 \%)$ & $29(23.8 \%)$ \\
Ativos & $42(14.8 \%)$ & $31(19.5 \%)$ & $11(9.0 \%)$ \\
\hline
\end{tabular}

Nota: * Associação significativa entre nível de atividade física e sexo $\left(\chi^{2}=6.52, d f=2, p=.038, \mathrm{~V}=\right.$ $.152)$

Tabela 2

Estresse (ISP) de bancários da amostra geral, homens e mulheres

\begin{tabular}{lcccc}
\hline & $M$ & $D P$ & Min & Max \\
\hline Amostra Geral & 23.15 & 6.15 & 10 & 44 \\
Homens & 23.16 & 6.21 & 11 & 44 \\
Mulheres & 23.14 & 6.11 & 10 & 42 \\
\hline
\end{tabular}


Saúde

Os bancários têm boa avaliação da saúde, visto que a maioria a auto-avalia como boa ou excelente. Os bancários também se mostraram pouco acometidos por doenças, sendo que a maior parte afirmou não ficar doente ou ficar doente poucas vezes. Homens e mulhe- res se diferenciaram significativamente apenas quanto à frequência de adoecimento, sendo os homens menos acometidos. A associação entre a frequência de adoecimento e o sexo foi fraca, de acordo com o coeficiente V (tabela $3)$.

Tabela 3

Auto-avaliação da saúde e frequência de adoecimento de bancários da amostra geral, homens e mulheres

\begin{tabular}{|c|c|c|c|c|c|c|}
\hline & \multicolumn{2}{|c|}{ Amostra Geral } & \multicolumn{2}{|c|}{ Homens } & \multicolumn{2}{|c|}{ Mulheres } \\
\hline & $n$ & $\%$ & $n$ & $\%$ & $n$ & $\%$ \\
\hline \multicolumn{7}{|c|}{ Auto-Avaliação da Saúde } \\
\hline Excelente & 33 & 11.7 & 21 & 13.2 & 11 & 9.0 \\
\hline Boa & 200 & 70.7 & 116 & 73.0 & 83 & 68.0 \\
\hline Regular & 47 & 16.6 & 22 & 13.8 & 25 & 20.5 \\
\hline Ruim & 3 & 1.0 & - & 一 & 3 & 2.5 \\
\hline Total & 283 & 100.0 & 159 & 100.0 & 122 & 100.0 \\
\hline \multicolumn{7}{|c|}{ Frequência de Adoecimento * } \\
\hline Não fica doente & 56 & 19.9 & 35 & 22.1 & 21 & 17.3 \\
\hline Fica poucas vezes & 189 & 67.3 & 109 & 69.0 & 78 & 64.5 \\
\hline Fica às vezes & 30 & 10.7 & 14 & 8.9 & 16 & 13.2 \\
\hline Fica muitas vezes & 6 & 2.1 & - & 一 & 6 & 5.0 \\
\hline Total & 281 & 100.0 & 158 & 100.0 & 121 & 100.0 \\
\hline
\end{tabular}

\section{Relação entre as principais variáveis em estudo}

Os resultados mostram que os bancários que praticam mais atividades físicas são menos estressados (tabela $4)$. Verifica-se diferença significativa ( $F$ $=4.87, p=.008$ ) na comparação do nível de estresse dos grupos com diferentes níveis de atividade física, sendo que o teste Post Hoc de Scheffé indicou diferença apenas entre os grupos ativo e inativo $(p=.039)$. Investigando separadamente homens e mulheres, percebe-se que somente as mulheres com diferentes níveis de atividade física se diferenciaram significativamente quanto ao estresse $(F=4.11, p=.019)$. O resultado do Post Hoc de Scheffé indicou diferença entre o grupo das bancárias ativas e o das inativas $(p=.028)$. 
Tabela 4

Estresse (ISP) em função do nível de atividade física de bancários da amostra geral, homens e mulheres

\begin{tabular}{lcccccc}
\hline & \multicolumn{2}{c}{ Amostra Geral * } & \multicolumn{2}{c}{ Homens } & \multicolumn{2}{c}{ Mulheres ** } \\
& $M$ & $D P$ & $M$ & $D P$ & $M$ & $D P$ \\
\hline Inativos & 24.04 & 6.23 & 24.06 & 6.41 & 24.02 & 6.10 \\
Moderadamente Ativos & 22.00 & 5.95 & 21.82 & 5.93 & 22.25 & 6.08 \\
Ativos & 21.36 & 5.55 & 22.26 & 5.73 & 18.82 & 4.29 \\
\hline
\end{tabular}

Nota: * Diferenças significativas no ISP de ativos, moderadamente ativos e inativos da amostra geral ao nível de $p<.01$; ** Diferenças significativas no ISP das bancárias ativas, moderadamente ativas e inativas ao nível de $p<.05$

Quanto à auto-avaliação da saúde dos bancários com diferentes níveis de atividade física, verificou-se que os mais ativos foram os que autoavaliaram a saúde mais positivamente (tabela 5). Percebeu-se associação positiva moderada, de acordo com o coefi- ciente Gamma, entre auto-avaliação da saúde e nível de atividade física. Por outro lado, diferenciando homens e mulheres, percebeu-se que essa associação foi significativa apenas para os homens (tabela 5).

Tabela 5

Auto-avaliação da saúde e frequência de adoecimento de bancários da amostra geral, homens e mulheres com diferentes níveis de atividade física

\begin{tabular}{|c|c|c|c|c|c|c|c|c|c|}
\hline & \multicolumn{3}{|c|}{ Amostra Geral } & \multicolumn{3}{|c|}{ Homens } & \multicolumn{3}{|c|}{ Mulheres } \\
\hline & A & MA & I & A & MA & I & A & MA & I \\
\hline & $\%$ & $\%$ & $\%$ & $\%$ & $\%$ & $\%$ & $\%$ & $\%$ & $\%$ \\
\hline \multicolumn{10}{|c|}{ Auto-Avaliação da Saúde * } \\
\hline Excelente & 26.2 & 16.2 & 6.4 & 25.8 & 17.9 & 6.7 & 27.3 & 13.8 & 4.9 \\
\hline Boa & 69.0 & 76.5 & 68.8 & 71.0 & 79.5 & 70.8 & 63.6 & 72.4 & 67.1 \\
\hline Regular & 4.8 & 7.3 & 23.1 & 3.2 & 2.6 & 22.5 & 9.1 & 13.8 & 24.4 \\
\hline Ruim & - & - & 1.7 & - & - & - & - & - & 3.6 \\
\hline Total & 100.0 & 100.0 & 100.0 & 100.0 & 100.0 & 100.0 & 100.0 & 100.0 & 100.0 \\
\hline \multicolumn{10}{|c|}{ Frequência de Adoecimento ** } \\
\hline Não fica doente & 33.3 & 26.9 & 14.0 & 35.5 & 30.8 & 13.6 & 27.3 & 21.4 & 14.6 \\
\hline Fica poucas vezes & 61.9 & 61.2 & 70.9 & 61.3 & 56.4 & 77.3 & 63.6 & 67.9 & 63.5 \\
\hline Fica às vezes & 4.8 & 11.9 & 11.6 & 3.2 & 12.8 & 9.1 & 9.1 & 10.7 & 14.6 \\
\hline Fica muitas vezes & - & - & 3.5 & - & - & - & - & 一 & 7.3 \\
\hline Total & 100.0 & 100.0 & 100.0 & 100.0 & 100.0 & 100.0 & 100.0 & 100.0 & 100.0 \\
\hline
\end{tabular}

Nota: A = Ativos; MA = Muito Ativos; I = Inativos; * Associação significativa entre auto-avaliação da saúde e nível de atividade física da amostra geral ao nível de $p=.000\left(\chi^{2}=26.77\right.$, $\left.d f=6, \gamma=.56\right)$ e homens ao nível de $p=.001\left(\chi^{2}=18.41, d f=4, \gamma=.60\right) ;{ }^{* *}$ Associação significativa entre frequência de adoecimento e nível de atividade física da amostra geral ao nível de $p=.022\left(\chi^{2}=14.80, d f=6, \gamma=\right.$ -.34) e homens ao nível de $p=.033\left(\chi^{2}=10.52\right.$, $\left.d f=4, \gamma=-.33\right)$ 
Bancários ativos demonstraram menor ocorrência de doenças quando comparados a bancários com menores níveis de atividade física (tabela 5). Estas variáveis mostraram-se associadas negativamente, de acordo com o coeficiente Gamma, em níveis moderados. Mais uma vez homens e mulheres se diferenciaram, pois tal associação só foi significativa para os homens (tabela $5)$.

Quanto mais negativa a percepção da saúde dos bancários, maior o ISP destes $(F=13.45, p=.000)$ (tabela 6$)$. O resultado do Post Hoc de Scheffé apresentou diferenças significativas entre $\mathrm{O}$ ISP dos bancários que avaliaram a saúde como ruim e excelente $(p=.009)$, ruim e boa $(p=.039)$, regular e boa $(p$ $=.000)$, e regular e excelente $(p=$ .000). As diferenças de ISP dos diferentes grupos de auto-avaliação da saúde foram significativas tanto para os homens $(F=9.75, p=.000)$ quanto para as mulheres $(F=7.88, p=.000)$. Entre os homens os grupos que se diferenciaram foram: regular e excelente saúde $(p=.001)$, e regular e boa saúde $(p=.001)$. Entre as mulheres se diferenciaram os grupos: ruim e excelente saúde $(p=.007)$, ruim e boa saúde ( $p$ $=.032)$, regular e excelente saúde $(p=$ $.008)$, e regular e boa saúde $(p=.018)$.

Comparando o ISP dos bancários que indicaram diferentes frequências de adoecimento (tabela 6), verificou-se que os grupos diferenciaram-se significantemente, sendo mais estressados aqueles que ficam doentes com maior frequência $(F=5.40, p=.001)$. Diferenças foram encontradas entre os que não ficam doente e os que ficam muitas vezes $(p=.004)$, e os que não ficam doente e os que ficam doentes às vezes $(p=.017)$.

Tabela 6

Estresse (ISP) em função da auto-avaliação da saúde e frequência de adoecimento de bancários da amostra geral, homens e mulheres

\begin{tabular}{lcccccc}
\hline & \multicolumn{2}{c}{ Amostra Geral } & \multicolumn{2}{c}{ Homens } & \multicolumn{2}{c}{ Mulheres } \\
& $M$ & $D P$ & $M$ & $D P$ & $M$ & $D P$ \\
\hline $\begin{array}{l}\text { Auto-Avaliação da Saúde * } \\
\quad\end{array}$ & & & & & & \\
$\quad$ Excelente & 20.33 & 5.34 & 20.48 & 5.61 & 19.27 & 4.43 \\
$\quad$ Boa & 22.53 & 5.95 & 22.73 & 6.11 & 22.30 & 5.76 \\
$\quad$ Regular & 27.17 & 5.23 & 27.95 & 4.85 & 26.48 & 5.54 \\
$\quad$ Ruim & 32.33 & 7.50 & - & - & 32.33 & 7.51 \\
Frequência de Adoecimento ** & & & & & & \\
$\quad$ Não fica doente & 21.54 & 5.79 & 21.34 & 6.02 & 21.86 & 5.52 \\
$\quad$ Fica poucas vezes & 23.13 & 5.79 & 23.51 & 5.79 & 22.58 & 5.78 \\
$\quad$ Fica às vezes & 24.53 & 7.70 & 24.50 & 9.04 & 24.56 & 6.63 \\
$\quad$ Fica muitas vezes & 31.17 & 6.34 & - & - & 31.17 & 6.34 \\
\hline
\end{tabular}

Nota: * Diferenças significativas no ISP de bancários da amostra geral, homens e mulheres com diferentes auto-avaliações da saúde ao nível de $p<.001 ;{ }^{* *}$ Diferenças significativas no ISP de bancários da amostra geral e mulheres com diferentes frequências de adoecimento ao nível de $p<.01$ 
Realizando as mesmas comparações, e dividindo homens e mulheres, percebeu-se que as diferenças nos níveis de estresse foram significativas apenas para as mulheres com frequências de adoecimento distintas $(F=$ $4.60, p=.004)$. As diferenças ocorreram entre as que ficam doentes muitas vezes e poucas vezes $(p=.010)$, e entre as que ficam doente muitas vezes e as que não ficam doente $(p=.011)$.

\section{DISCUSSÃO}

O presente estudo objetivou investigar a relação entre o nível de atividade física, o estresse e a saúde de bancários e bancárias. Buscou-se verificar se a relação entre tais variáveis são equivalentes para ambos os sexos.

Os bancários mostram-se mais fisicamente ativos do que as bancárias. Esse resultado vai ao encontro de outros estudos que investigaram $o$ nível de atividade física em populações de trabalhadores brasileiros (Barros \& Nahas, 2001). Quanto à população bancária, Andrade (2001) verificou que cerca de $60 \%$ dos bancários (homens) de um banco estatal de Florianópolis eram sedentários. Em estudo semelhante, Viana, Vasconcellos e Fonseca (2006), pesquisando bancárias da mesma região, verificaram que as mulheres deste meio eram ainda mais sedentárias, pois $73 \%$ não praticavam atividade física. Essa realidade parece ter caráter social, tendo relação com o tratamento diferenciado dado desde a infância a meninos e meninas, o que acaba se refletindo por toda a vida. Enquanto mulheres têm preferência por atividades individuais e que exigem menos esforço, os homens preferem atividades coletivas com maior desgaste físico (Salles-Costa, Werneck, Lopes, \& Faerstein, 2003).

Analisando os níveis de estresse apresentado pelos bancários, percebese que o mesmo é ligeiramente elevado. Por outro lado, considerando a especificidade da população e comparando com estudos brasileiros mais recentes que utilizaram o mesmo instrumento com esta população (Andrade, 2001; Viana et al., 2006), os bancários do presente estudo apresentaram menores níveis de estresse.

Até a década de 1970 os efeitos do estresse eram investigados preferencialmente em homens, pois se acreditava que os ciclos hormonais das mulheres prejudicariam os estudos (Lundberg, 2005). Atualmente, as pesquisas têm verificado que as mulheres apresentam um maior nível de estresse em comparação aos homens (Barros \& Nahas, 2001; Lundberg, \& Frankenhaeuser, 1999; Wijndaele et al., 2007). Essas diferenças parecem não estar condicionadas apenas a fatores biológicos, mas têm também relação com os papéis sociais de homens e mulheres. No estudo de Lundberg e Frankenhaeuser (1999), com homens e mulheres trabalhadores cujas funções são semelhantes as dos bancários, foi verificado que os níveis de adrenalina durante a jornada de trabalho de ambos os sexos foram semelhantes, pouco maior entre as mulheres. Mas o resultado mais importante ocorreu após o fim da jornada de trabalho, quando os homens reduziram seus níveis de adrenalina mais rapidamente, e as mulheres 
mantiveram-se com elevados níveis por um maior tempo. Os autores apontaram a hipótese de que a preocupação com o cuidado dos filhos e o trabalho doméstico (dupla jornada) que ocorre após o trabalho foram os responsáveis pela permanência do elevado nível de adrenalina nas mulheres.

Pode-se discutir a ocorrência de estresse semelhante entre os sexos no presente estudo em função das considerações de Dedecca (2004). Para o referido autor, as mulheres que sofrem com a dupla jornada são aquelas que recebem baixos salários e não apresentam condições de pagar alguém que ajude de alguma forma em seu trabalho doméstico. Nesse sentido as bancárias poderiam ser beneficiadas, pois apresentam salários superiores à média das trabalhadoras brasileiras, sofrendo menos com a dupla jornada.

A avaliação da saúde dos bancários foi positiva. Homens e mulheres não se diferenciaram significativamente quando à percepção da saúde, porém os homens apresentaram menor frequência de adoecimento. Esse resultado corrobora com outros estudos realizados no Brasil, inclusive pesquisas que envolveram a população bancária (Silva et al., 2007).

Aquino et al. (1992) apresentam resultados da Pesquisa Nacional por Amostra de Domicílios, que evidenciam que as mulheres são mais susceptíveis a enfermidades que os homens, especialmente na idade produtiva, idade em que se encontram os bancários desta pesquisa. O estudo foi realizado levando em consideração os casos de doenças que levam a procura de aten- dimento médico. Desta forma, os autores sugerem que diferenças de gênero na construção da experiência de adoecimento, tanto na percepção, quanto no relato diferenciado entre os sexos, contribuem para a existência de diferenciais no adoecimento e utilização de serviços de saúde. Assim, os homens poderiam estar se dirigindo menos aos serviços de saúde não apenas por serem menos acometidos por doenças, mas também devido a terem uma melhor percepção de sua saúde em comparação às mulheres. Isso mostra a relevância de pesquisas com caráter percebido da saúde.

Em pesquisa que avaliou a saúde de forma semelhante a do presente estudo, verificou-se que as diferenças na saúde de homens e mulheres se alteravam para as diferentes raças; mesmo assim os homens apresentaram uma melhor percepção da saúde para todas (Read \& Gorman, 2006). McDonough e Walters (2001) afirmam que a ideia de que as mulheres têm mais problemas de saúde que os homens é sustentada empiricamente, mas que as diferenças são mais complexas do que os estudos vêm se propondo a investigar, suscitando a investigação de outras variáveis, como o estresse e a atividade física.

Apesar de algumas pesquisas não apontarem de forma clara a relação "estresse e atividade física", fica a evidência de que a atividade física é um fator positivo no auxílio da redução do estresse. Mesmo quando os resultados são pouco significativos, estudos com diversas populações têm sugerido que o nível de estresse dos ativos é inferior ao 
dos inativos fisicamente (Andrade, 2001; Kouvonen et al., 2005). Não foram localizadas pesquisas mostrando o contrário, o que aponta que mesmo quando a redução do estresse em função da atividade física é pequena, ela existe para homens e mulheres.

Percebeu-se no presente estudo diferença significativa entre os níveis de estresse dos bancários ativos e inativos. Observando separadamente bancários homens e mulheres, essas diferenças foram significativas apenas para as mulheres. Esse resultado contradiz pesquisas anteriores realizadas com bancários brasileiros, que indicavam o contrário: diferença significativa entre homens (Andrade, 2001) e não significativa entre as mulheres (Viana et al., 2006).

Se por um lado essa discordância entre os resultados prejudica a compreensão das relações entre sexo, atividade física e estresse, por outro lado a questão de diferenciar os sexos nessa comparação se fortalece. Estudos têm mostrado que a forma de enfrentamento do estresse e as estratégias para reduzi-lo diferenciam-se entre homens e mulheres (González-Morales, Peiró, Rodríguez, \& Greenglass, 2006). Enquanto a sustentação social mostrase mais benéfica para a redução do estresse das mulheres, entre os homens a melhor maneira de lidar com o estresse é agindo direto sobre o agente estressor (González-Morales et al., 2006). Esses achados reforçam a ideia de que a influência da atividade física sobre os níveis de estresse de homens e mulheres pode ser diferente, pois suas estratégias de enfrentamento também o são.

A prática de atividade física também está associada à saúde, sendo as pessoas ativas mais saudáveis ( $\mathrm{Hu}$ et al., 2004; Kouvonen et al., 2005; WHO, 2002). Essa associação existente entre uma boa saúde e a prática de atividade física também foi observada no presente estudo. Os bancários mais ativos auto-avaliam melhor sua saúde e percebem menor frequência de adoecimento quando comparados aos bancários inativos.

Como os benefícios que a prática de atividade física proporciona para o seu praticante não são apenas físicos, mas também psicológicos, é relevante a avaliação subjetiva da saúde. Quanto à qualidade de vida, pouco adiantaria um check-up que exibe excelente saúde, se o indivíduo estiver, no fundo, se sentindo mal. Então, a prática de atividade física pode ser um fator que auxilie na melhora da auto-imagem do indivíduo, fazendo com que o mesmo sinta-se saudável. Essa afirmação é amparada por Ransford e Palisi (1996), que consideram que a prática de uma atividade faz com que o indivíduo sinta-se menos vulnerável a doenças, principalmente quando a prática é realizada com o objetivo de melhorias na saúde.

Considerando que as mulheres do presente estudo apresentaram pior saúde do que os homens, esperava-se que as mesmas fossem mais beneficiadas pela prática de atividades físicas, mas essas expectativas não foram confirmadas. A melhoria da percepção da saúde e adoecimento esteve mais relacionada com a prática de atividade físi- 
ca para os homens, enquanto para as mulheres essa relação não foi significativa. Quando Ransford e Palisi (1996) investigaram as diferenças na relação entre a saúde percebida e prática de atividade física de homens e mulheres, haviam criado a hipótese de que as mulheres seriam mais beneficiadas com a prática, mas essa hipótese não foi confirmada consistentemente por sua pesquisa. Dentre quatro grupos praticantes de atividade física (caminhada, natação, dança e corrida), apenas se diferenciaram os homens e mulheres que praticavam caminhada, mais válida para as mulheres, e corrida, mais válida para os homens.

A presença de problemas de saúde parece estar associado com níveis elevados de estresse (Lundberg, 2005). Essa relação parece bem estabelecida, corroborando com nossos resultados, onde os bancários que auto-avaliam a saúde mais positivamente são menos estressados. Devido às características da presente pesquisa, existe a limitação de não sabermos se é o estresse que provoca uma maior frequência de adoecimento nos bancários ou se são as doenças que elevam os níveis de estresse dessa população; mas parece clara, segundo nossos resultados, a associação entre essas variáveis.

Pesquisadores acreditam que ainda são limitadas as pesquisas na área de "atividade física e saúde" (Plotnikoff et al., 2004), sendo necessários estudos que investiguem diferentes grupos, para que se possa intervir de maneira mais específica e efetiva. É necessário que se tenha uma visão mais crítica sobre a realidade e se ponha em ques- tão nessa área também temas já considerados "resolvidos". Sugerem-se novos estudos que destaquem não apenas as diferenças entre os sexos, mas também entre diferentes idades, população urbana e rural, classe econômica, dentre outras.

\section{CONCLUSÕES}

O presente estudo indicou a existência de relações entre o nível de atividade física, o estresse e a saúde de bancários. Separando as análises por sexo, observou-se as seguintes diferenças entre bancários e bancárias: a) A prática de atividade física está associada com menores níveis de estresse apenas para as bancárias; b) $\mathrm{O}$ nível de atividade física esteve associado com a percepção da saúde e frequência de adoecimento, sendo mais saudáveis os bancários mais ativos, porém os resultados só foram significativos para os homens; e, c) Grupos com pior percepção da saúde são mais estressados, tanto para os homens quanto para as mulheres, porém a frequência de adoecimento só esteve associada com o nível de estresse das mulheres. Estes resultados indicam a necessidade do controle da variável sexo quando tais variáveis forem investigadas, e demonstram que as relações entre estas são complexas e ainda não conclusivas.

É importante considerar que a população de trabalhadores investigada nesta pesquisa é bastante específica, tendo características peculiares por seu tipo de ofício. Abordar novas possibilidades de estudos em outras regiões ou populações de trabalhadores é válido, pela possibilidade de se encontrar 
novos resultados que auxiliem no entendimento da problemática ora investigada.

Considerando que a população dos bancários é uma das mais acometidas pelo estresse, e que este tem implicações negativas à saúde, a presente pesquisa contribui na soma de informações aos envolvidos nesse tipo de trabalho ao apontar a atividade física como uma possível ferramenta para uma saúde mais positiva. Desta forma, levandose em conta o baixo nível de atividade física da amostra investigada, programas que favoreçam a adoção de um estilo de vida mais saudável, incluindo a prática de exercícios físicos, podem ser benéficos para uma melhor qualidade de vida para essa população.

\section{REFERÊNCIAS}

Adami, F., Frainerm, D. E. S., Santos, J. S., Fernandes, T. C., \& De-Oliveira, F. R. (2008). Insatisfação corporal e atividade física em adolescentes da região continental de Florianópolis. Psicologia: Teoria e Pesquisa, 24(2), 143-149.

Andrade, A. (2001). Ocorrência e controle subjetivo do stress na percepção de bancários ativos e sedentários: a importância do sujeito na relação "atividade física e saúde". Tese de doutorado, Universidade Federal de Santa Catarina, Florianópolis, Santa Catarina, Brasil.

Aquino, E. M. L., Menezes, G. M. S., \& Amoedo, M. B. (1992). Gênero e saúde no Brasil: Considerações a partir da pesquisa nacional por amostra de domicílios. Revista de Saúde Pública, 26(3), 195-202.

Barros, M. V. G., \& Nahas M. V. (2001). Comportamentos de risco, autoavaliação do nível de saúde e percepção de estresse entre trabalhadores da indústria. Revista de Saúde Pública, 35(6), 554-563.

Calais, S. L., Andrade, L. M. B., \& Lipp, M. E. N. (2003). Diferenças de sexo e escolaridade na manifestação de stress em adultos jovens. Psicologia: Reflexão e Crítica, 16(2), 257-263.

Cohen, S., Karmack, T., \& Mermelsteinm, R. (1983). A global measure of perceived stress. Journal of Health and Social Behavior, 24(4), 385-396.

Dedecca, C. S. (2004). Tempo, trabalho e gênero. Acedido em Setembro, 28 de 2008 a partir de http://www.ie.ufrj.br/aparte/pdfs/not a_tecnica_tempo_trabalho_e_genero.p df.

França A. C. L., \& Rodrigues, A. L. (1997). Stress e trabalho: Guia básico com abordagem psicossomática. São Paulo: Atlas

Glaner, M. F. (2007). Concordância de questionários de atividade física com a aptidão cardiorrespiratória. Revista Brasileira de Cineantropometria \& Desempenho Humano, 9(1), 61-66.

González-Morales, M. G., Peiró, J. M., Rodríguez, I., \& Greenglass, E. R. (2006). Coping and distress in organizations: The role of gender in work stress. International Journal of Stress Management, 13(2), 228-248.

Hu, G., Eriksson, G. H. J., Barengo, N. C., Lakka, T. A., Valle, T. T., Nissinen A., et al. (2004). Occupational, commuting, and leisure-time physical activity in relation to total and cardiovascular mortality among Finnish subjects with type 2 diabetes. Circulation, 110(6), 666-673.

Kouvonen, A., Kivimäki, M., Elovainio, M., Virtanen, M., Linna, A., \& Vahtera, J. (2005). Job strain and leisure-time physical activity in female and male public sector employees. Preventive Medicine, 41 (2), 532-539. 
Lundberg, U. (2005). Stress hormones in health and illness: The roles of work and gender. Psychoneuroendocrinology, 30(10), 1017-1021.

Lundberg, U., \& Frankenhaeuser, M. (1999). Stress and workload of men and women in high-ranking positions. Journal of Occupational Health Psychology, 4(2), 142-151.

Matud, M. P. (2004). Gender differences in stress and coping styles. Personality and Individual Differences, 37(7), 1401-1415.

McDonough, P., \& Walters V. (2001). Gender and health: Reassessing patterns and explanations. Social Science $\mathcal{E}$ Medicine, 52(4), 547-559.

Nahas, M. V. (2001). Atividade física, saúde e qualidade de vida: Conceitos e sugestões para um estilo de vida ativo. Londrina: Midiograf.

Palácios, M., Duarte, F., \& Câmara, V. M. (2002). Trabalho e sofrimento psíquico de caixas de agências bancárias na cidade do Rio de Janeiro. Cadernos de Saúde Pública, 18(3), 843-851.

Paschoal, T., \& Tamayo, A. (2004). Validação da escala de estresse no trabalho. Estudos em Psicologia, 9(1), 45-52.

Pate, R. R., Pratt, M., Blair, S. N., Haskel, W. L., Macera, C. A., Bouchard, C., et al. (1995). Physical activity and public health: A recommendation from the Centers for Disease Control and Prevention and the American College of Sports Medicine. The Journal of the American Medical Association, 273, 402-407.

Plotnikoff, R. C., Mayhew, A., Birkett, N., Loucaides, C. A., \& Fodor, G. (2004). Age, gender, and urban-rural differences in the correlates of physical activity. Preventive Medicine, 39(6), 11151125.

Ransford, H. E., \& Palisi, B. J. (1996). Aerobic exercise, subjective health and psychological well-being within age and gender subgroups. Social Science \& Medicine, 42 (11), 1555-1559.

Read, J. G., \& Gorman, B. K. (2006). Gender inequalities in US adult health: The interplay of race and ethnicity. Social Science \& Medicine, 62 (12), 1045-1065.

Rocha, L. E., \& Debert-Ribeiro, M. (2001). Trabalho, saúde e gênero: Estudo comparativo sobre analistas de sistemas. Revista de Saúde Pública, 35(6), 539-547.

Salles-Costa, R., Werneck, G. L., Lopes, C. S, \& Faerstein, E. (2003). Associação entre fatores sócio-demográficos e prática de atividade física de lazer no Estudo Pró-Saúde. Cadernos de Saúde Pública, 19(S2), 1095-1105.

Silva, L. S., Pinheiro, T. M. M., \& Sakurai, E. (2007). Reestruturação produtiva, impactos na saúde e sofrimento mental: O caso de um banco estatal em Minas Gerais, Brasil. Cadernos de Saúde Pública, 23(12), 2949-2958.

Souza, M. F. M., Messing, K., Menezes, P. R., \& Cho, H. J. (2002). Chronic fatigue among bank workers in Brazil. Occupational Medicine, 52 (4), 187-194.

Ururahy, G. (1997). Como tornar-se um bom estressado. Rio de Janeiro: Salamandra.

Viana, M. S., Vasconcellos, D. I. C., \& Fonseca, A. B. P. (2006). Influence of the life style on stress perception of active and sedentary bank clerks. FIEP Bulletin, 73, 396-399.

World Health Organization (2002). The world health report: Reducing risks, promoting healthy life. Geneva: Autor.

Wijndaele, K., Matton, L., Duvigneaud, N., Lefevre, J., Bourdeaudhuij, I. D., Duquet, W., et al. (2007). Association between leisure time physical activity and stress, social support and coping: A cluster-analytical approach. Psychology of Sport and Exercise, 8(4), 425-440. 